\title{
Guest Editorial: Visual Multimedia Learning from Big Surveillance Data
}

\author{
Zheng $\mathrm{Xu}^{1} \cdot$ Junchi $\mathrm{Yan}^{2} \cdot$ Richard Yida $\mathrm{Xu}^{3} \cdot \mathrm{Lin} \mathrm{Mei}^{1}$
}

Published online: 23 May 2017

(C) Springer Science+Business Media New York 2017

Intelligent video surveillance is an important topic in the field of computer vision and pattern recognition. Significant progress has achieved in the last decades, from object detection, tracking and parsing to activity recognition and video understanding. Visual multimedia learning, which can be treated as the most significant breakthrough in past 10 years, has greatly affected the methodology of computer vision and achieved terrific progress in both academy and industry. Visual multimedia learning is firstly adopted in ImageNet Competition for object categorization, which achieved a 12\% progress in 2012 and confirmed the priority of deep learning for computer vision applications. From then on, deep learning has been adopted in all kinds of computer vision applications and many breakthroughs have achieved in subareas, like DeepFace on LFW competition for face verification, GoogleNet for ImageNet Competition for object categorization. It can be expected that more and more computer vision applications will benefit from Visual multimedia learning.

The 56 submitted manuscripts were reviewed by experts from both academia and industry. After two rounds of reviewing, 34 manuscripts were accepted for this special issue.

Acknowledgements The guest editors would like to thank Prof. Borivoje Furht who is the editor in chief of Multimedia Tools and Applications. His help and trust is the most important thing for the success of this SI. The guest editors would like to thank the reviewers for their high quality reviews, which provided insightful and constructive feedback to the authors of the papers.

Zheng Xu

juven_xz@163.com

1 The Third Research Institute of the Ministry of Public Security (TRIPMS), Shanghai, China

2 IBM Research, Beijing, China

3 University of Technology Sydney, Sydney, Australia 